\title{
A network approach to response inhibition: dissociating functional connectivity of neural components involved in action restraint and action cancellation
}

Citation for published version (APA):

Dambacher, F., Sack, A. T., Lobbestael, J., Arntz, A., Brugman, S., \& Schuhmann, T. (2014). A network approach to response inhibition: dissociating functional connectivity of neural components involved in action restraint and action cancellation. European Journal of Neuroscience, 39(5), 821-831.

https://doi.org/10.1111/ejn.12425

Document status and date:

Published: 01/03/2014

DOI:

10.1111/ejn.12425

Document Version:

Publisher's PDF, also known as Version of record

Document license:

Taverne

Please check the document version of this publication:

- A submitted manuscript is the version of the article upon submission and before peer-review. There can be important differences between the submitted version and the official published version of record. People interested in the research are advised to contact the author for the final version of the publication, or visit the DOI to the publisher's website.

- The final author version and the galley proof are versions of the publication after peer review.

- The final published version features the final layout of the paper including the volume, issue and page numbers.

Link to publication

\footnotetext{
General rights rights.

- You may freely distribute the URL identifying the publication in the public portal. please follow below link for the End User Agreement:

www.umlib.nl/taverne-license

Take down policy

If you believe that this document breaches copyright please contact us at:

repository@maastrichtuniversity.nl

providing details and we will investigate your claim.
}

Copyright and moral rights for the publications made accessible in the public portal are retained by the authors and/or other copyright owners and it is a condition of accessing publications that users recognise and abide by the legal requirements associated with these

- Users may download and print one copy of any publication from the public portal for the purpose of private study or research.

- You may not further distribute the material or use it for any profit-making activity or commercial gain

If the publication is distributed under the terms of Article 25fa of the Dutch Copyright Act, indicated by the "Taverne" license above,

Download date: 26 Apr. 2023 


\title{
A network approach to response inhibition: dissociating functional connectivity of neural components involved in action restraint and action cancellation
}

\author{
Franziska Dambacher, ${ }^{1,2}$ Alexander T. Sack, ${ }^{1,2}$ Jill Lobbestael, ${ }^{3}$ Arnoud Arntz, ${ }^{3}$ Suzanne Brugman ${ }^{3}$ and Teresa \\ Schuhmann ${ }^{1,2}$ \\ ${ }^{1}$ Department of Cognitive Neuroscience, Faculty of Psychology and Neuroscience, Maastricht University, Oxfordlaan 55, \\ $6229 \mathrm{EV}$, Maastricht, The Netherlands \\ ${ }^{2}$ Maastricht Brain Imaging Center, Maastricht, The Netherlands \\ ${ }^{3}$ Department of Clinical Psychological Science, Maastricht University, Maastricht, The Netherlands
}

Keywords: functional magnetic resonance imaging, go/no-go, human, inferior frontal cortex, response inhibition, stop signal

\begin{abstract}
The ability to inhibit action tendencies is vital for adaptive human behaviour. Various paradigms are supposed to assess action inhibition and are often used interchangeably. However, these paradigms are based on different conceptualizations (action restraint vs. action cancellation) and the question arises as to what extent different conceptualizations of inhibitory processing are mirrored in a distinct neural activation pattern. We used functional magnetic resonance imaging to investigate the neural correlates of action restraint vs. action cancellation. Analyses of local activity changes as well as network connectivity measures revealed a strong overlap of activation within a common action inhibition network including inferior frontal, pre-supplementary motor and thalamic brain areas as well as the anterior cingulate cortex. Furthermore, our findings pointed to additional neural networks that are distinct for action restraint (i.e. right superior frontal gyrus, left middle frontal gyrus, and bilateral anterior cingulate cortex) and action cancellation (i.e. right middle frontal gyrus, posterior cingulate cortex, and parietal regions). Our connectivity analyses showed that different inhibitory modalities largely relied on a task-independent global inhibition network within the brain. Furthermore, they suggested that the conceptually distinct inhibitory aspects of action restraint vs. action cancellation also activated additional specific brain regions in a task-dependent manner. This has implications for the choice of tasks in an empirical setting, but is also relevant for various clinical contexts in which inhibition deficits are considered a diagnostic feature.
\end{abstract}

\section{Introduction}

Social interaction relies heavily on an actor's capacity to modify reactions and to inhibit pre-planned or automatic responses. Therefore, response (or action) inhibition, defined as the cognitive ability to withhold such reactions (Logan et al., 1997), is considered to be one of the key concepts in understanding the flexible and adaptive nature of human behaviour.

Various paradigms have been developed to assess response inhibition (Wöstmann et al., 2012), of which the go/no-go task (GNGT) and the stop-signal task (SST) are the most prominent. Both paradigms share the task instruction to not respond to a certain configuration of stimuli and might, thus, revert to similar cognitive resources (Schachar et al., 2007). However, both paradigms are understood to employ different aspects of inhibitory processing ( $\mathrm{Ru}-$ bia et al., 2001; Aron et al., 2004; McNab et al., 2008; Zheng et al., 2008; Swick et al., 2011). Eagle et al. (2008) stated that, in a

Correspondence: Franziska Dambacher, ${ }^{1}$ Department of Cognitive Neuroscience, as above.

E-mail: franziska.dambacher@maastrichtuniversity.nl

Received 29 July 2013, accepted 17 October 2013
GNGT, a not-yet-initiated action has to be restrained. In contrast, in an SST, already initiated responses have to be cancelled. Therefore, it is of interest to what extent such conceptual differences are mirrored by differences in task-related neural activity.

Brain imaging studies have consistently reported inhibition-related activity within mostly right-lateralized (Aron et al., 2004) ventral and inferior prefrontal cortex, pre-supplementary motor areas, and sub-cortical circuitries (Chambers et al., 2009). Only a few studies have directly compared neural activation in go/no-go and stop-signal paradigms, focusing mainly on overlapping activation and identifying bihemispheric inferior, right middle frontal, and left anterior insula regions (McNab et al., 2008; Zheng et al., 2008) as the common locus of inhibitory processing. Rubia et al. (2001) reported left-lateralized prefrontal and parietal activation for action restraint and more right hemispheric activation during action cancellation. Sebastian et al. (2013) disentangled common and specific neural sub-processes of different inhibitory aspects. They found empirical evidence for a common inhibition network including the inferior frontal cortex, pre-supplementary motor area, and parietal regions, but still emphasized that different aspects of action inhibition, i.e. interference inhibition and action cancellation, are discriminable at 
the neural level. In addition, Swick et al. (2011) proposed, in their comprehensive meta-analysis, that both tasks share a fundamental core network involving bilateral inferior frontal and pre-supplementary motor regions. Furthermore, they assumed an additional frontoparietal network to be involved in action restraint and a cinguloopercular network in action cancellation.

However, the identification of common and distinct inhibitory neural networks and their respective brain connectivity measures were not directly addressed in any of the above-mentioned studies. A connectivity approach is indispensable in order to enable the mere description of co-activation of brain regions to move towards an understanding of their actual interaction. Therefore, we designed a functional imaging study in order to look at connectivity as well as local activity changes during response inhibition. Action restraint was expected to activate a fronto-parietal network, whereas action cancellation was thought to activate a cingulo-opercular network. In addition to these task-dependent neural network patterns, we furthermore also expected to find a common right-lateralized inhibition network including inferior frontal and pre-supplementary motor areas.

\section{Materials and methods}

\section{Participants and procedure}

Nineteen healthy Dutch males volunteered for this study. One participant was excluded from further analysis because of technical problems during functional magnetic resonance imaging (fMRI) data acquisition and one participant did not complete the experiment. Therefore, the data of 17 participants (mean age 28.4 years, SD 9.9 years) were included in the data analysis. All participants gave their written informed consent for participation and were paid for taking part. The study was approved by the Ethical Commission of the Faculty of Psychology and Neuroscience at Maastricht University and conformed to the Code of Ethics of the World Medical Association (Declaration of Helsinki).

Participants had to complete two response inhibition tasks during the fMRI scan. Both task designs were as similar as possible in order to enable a valid comparison of their specific distinct inhibitory mechanisms. Furthermore, feasibility and comfortable task execution inside the fMRI scanner were considered to enable participants to perform with full concentration.

In order to elicit action restraint, a simple go/no-go motor response task was used (Fig. 1A). Participants were instructed to respond as fast and accurately as possible to a go stimulus via button press, whereas they should not respond to a rare no-go stimulus. Go as well as no-go stimuli were presented for $200 \mathrm{~ms}$ followed by a fixation cross for a randomized interval of 1300, 2800, or $4300 \mathrm{~ms}$.

In order to elicit action cancellation, a modified version of the SST (Logan et al., 1997) was used (Fig. 1B). Participants were instructed to respond as fast and accurately as possible to a go stimulus via button press, whereas they should not respond to this same stimulus in the rare cases when it was followed by a visual stop signal. In the go trials, the go stimulus was presented for $200 \mathrm{~ms}$ followed by a fixation cross for a randomized interval of 1300, 2800, or $4300 \mathrm{~ms}$. In the stop trials, the go stimulus was presented for $200 \mathrm{~ms}$ followed by a stimulus fixed-onset asynchrony of $250 \mathrm{~ms}$, a visual stop signal presented for $300 \mathrm{~ms}$ and subsequently a fixation cross for a randomized interval of 950,2450 , or $3950 \mathrm{~ms}$. The fixed stop-signal delay was chosen to ensure optimal comparability between both paradigms and to keep the difficulty of the task at a level at which enough inhibition trials could be completed success-

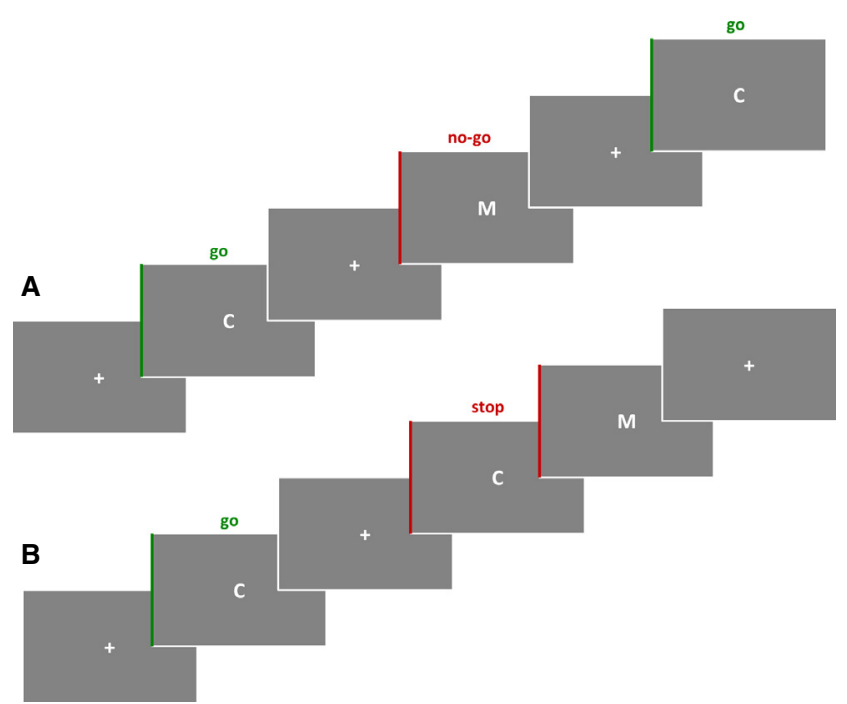

FIG. 1. (A) GNGT. Go trials shown in green, no-go/stop trials shown in red. (B) SST. Go trials shown in green, no-go/stop trials shown in red. In this example, $\mathrm{C}$ corresponds to the go stimulus in both tasks, while M corresponds to the no-go stimulus in the GNGT and the stop signal in the SST.

fully. It has to be emphasized that this task design did not allow the calculation of stop-signal reaction times within the framework of the independent race model (Verbruggen \& Logan, 2009).

No-go/stop events occurred in $25 \%$ of the 160 pseudo-randomized trials. The letters $\mathrm{C}$ and $\mathrm{M}$ were used as stimuli. The assignment of a letter to the go and no-go/stop condition was randomized between participants. After every 16 trials there was a short resting period of $19500 \mathrm{~ms}$ integrated into the task, when only a fixation cross was shown. Stimuli and fixation crosses were presented in white (RGB 255/255/255; Arial 24 point) on a grey background (RGB 125/125/ 125). Participants had to perform two consecutive runs of 160 trials for each task in randomized order, giving a total of 320 trials (80 no-go/stop trials) per task and a scanning time of about $1.5 \mathrm{~h}$ (approximately $10 \mathrm{~min}$ for each experimental run, plus acquisition of anatomical data, plus preparation). The task order was counterbalanced. After being instructed and before entering the fMRI scanner, all participants had completed a training version of each task (including 64 trials per task). Stimuli were presented using Presentation software (Neurobehavioural Systems, Inc., Albany, CA, USA).

\section{Technical details and functional magnetic resonance imaging acquisition}

Stimulus material was displayed on a frosted screen, positioned at the rear of the scanner bore, using an LCD projector (PLC-XT1116; Sanyo North American Corporation, San Diego, CA, USA). Responses were collected by employing a standard magnetic resonance-compatible button box (LUMItouch keypads, Photon Control, Burnaby, BC, Canada).

Using a $3 \mathrm{~T}$ Allegra magnetic resonance Scanner (Siemens), structural (high-resolution T1-weighted MPRAGE; isotropic voxel resolution $1 \times 1 \times 1 \mathrm{~mm}^{3}$; 192 sagittal slices) and functional (gradient-echo-sequence; repetition time, $1500 \mathrm{~ms}$; echo time, $28 \mathrm{~ms}$; field of view, $224 \mathrm{~mm}$; flip angle, $71^{\circ}$; matrix, $64 \times 64$; slice thickness, $3.5 \mathrm{~mm}$; distance factor, 10\%; 458 volumes per run) wholebrain images were acquired. A total of 27 oblique transverse slices of $3.5 \times 3.5 \times 3.5 \mathrm{~mm}$ voxels tilted $30^{\circ}$ relative to the anterior commissure-posterior commissure plane were obtained in order to avoid signal dropout in frontal areas (Deichmann et al., 2003). 


\section{Functional magnetic resonance imaging analysis}

Data analyses were performed using Brain Voyager QX 2.8 (Brain Innovation BV, Maastricht, Netherlands). The first five volumes of each functional run were discarded to allow for equilibrium effects. Pre-processing included three-dimensional motion correction (as implemented in Brain Voyager QX with trilinear/sinc interpolation and intrasession alignment to the first functional volume recorded after the individual anatomical scan), cubic spline slice scan time correction, and the application of a temporal high-pass filter [general linear model (GLM) with Fourier basis set of three cycles sine/ cosine per run including linear trend removal]. Images were co-registered to the individual anatomical scans and normalized to Talairach stereotaxic space (Talairach \& Tournoux, 1988). Volume time courses were spatially smoothed using a $6 \mathrm{~mm}$ full-width half-maximum Gaussian kernel.

In order to establish the activation patterns of the GNGT and SST as well as their dissociative brain activity, event-related randomeffects group analyses were performed. A GLM was defined in order to analyse specific task-related activation patterns for no-go trials in the GNGT and specific activation patterns for stop trials in the SST. The GLM included four predictors (successful go and successful nogo trials in the GNGT, and successful go and successful stop trials in the SST). Furthermore, commission errors on go trials (misses) and on no-go and stop trials (false alarms) were modeled in order to account for error variance. For each trial, blood oxygenation leveldependent responses during a time window of $1500 \mathrm{~ms}$ (one complete trial excluding the jitter) were modeled. Motion parameters were included as confound predictors in the regression analysis and the single regressors in the resulting matrix were tested in order to avoid multicollinearity. Statistical maps were created using a threshold of $P<0.01$ corrected for multiple comparisons by means of cluster threshold level estimation analysis of 1000 Monte Carlo simulation iterations (Forman et al., 1995), resulting in a cluster threshold of 28 voxels. A conjunction analysis for successful no-go and stop trials (no-go $\wedge$ stop) was conducted to statistically evaluate the overlap (and only the overlap) of voxels significantly activated during both tasks and therefore involved in both tasks. Furthermore, a direct contrast analysis (stop > no-go) was performed to directly compare the two tasks and to examine the dissociative brain activity patterns between the no-go trials in the GNGT and the stop trials in the SST.

\section{Connectivity analysis, choice and definition of seed regions}

In order to dissociate the overlapping and differential neural networks involved in both tasks, functional connectivity analyses were performed using instantaneous correlation mapping (Goebel et al., 2003; Roebroeck et al., 2005). We intended to investigate whether regions activated during action restraint and cancellation (in the GLM analyses) did indeed form a task-related functional network of orchestrated activity across distributed regions. This type of analysis requires the definition of seed regions. The task-related linear pairwise correlations were computed during the no-go and stop condition for each voxel between the average time course of the voxels in the seed region and the voxel time course.

The choice of seed regions was based on previous literature (Swick et al., 2011) and the concrete results of our GLM analysis. We focused on three potential networks: a supposedly common inhibition network, a specific inhibition network for action restraint, and a specific inhibition network for action cancellation. Therefore, connectivity analyses were based on three different seed regions (one for each network). In order to fulfill the criteria to be selected as seed regions, the regions had to be located within the hypothetical networks involved in action restraint and cancellation as suggested by Swick et al. (2011), and, furthermore, display significant neural activation in our GLM analysis. We chose the following seed regions:

- a common seed region in the right anterior insula (as the crucial node in a global inhibition network);

- an action restraint-specific seed region in the right frontal cortex (as the crucial node in an action restraint fronto-parietal network); and

- an action cancellation-specific seed region in the posterior cingulate cortex (PCC) (as the crucial node in an action cancellation cingulo-opercular network).

The regions of interest (ROIs) within these seed regions were defined depending on the specific group random-effects GLM activation, focusing on the 250-300 most significantly activated voxels in the three regions. The ROI on which the connectivity analysis regarding a potential common inhibition system was based was defined on the GLM map of the conjunction between successful action restraint and action cancellation (no-go $\wedge$ stop). The ROI on which the connectivity analysis regarding a potential action restraint-specific inhibition system was based was defined on the GLM map of successful action restraint vs. successful action cancellation (no-go > stop). The ROI on which the connectivity analysis regarding a potential action cancellation-specific inhibition system was based was defined on the GLM map of successful action cancellation vs. successful action restraint (stop $>$ no-go).

Group-level instantaneous correlation maps were computed by averaging all single-subject maps and testing the average $T>0$. Cluster threshold level estimation analysis was performed with $P<0.0001$ and 1000 Monte Carlo simulation iterations in order to correct for multiple comparisons (Forman et al., 1995), resulting in a cluster threshold of five voxels.

\section{Results}

\section{Behavioural data}

Participants reacted significantly faster in the successful go trials for the GNGT compared with the SST (GNGT: mean $412.5 \mathrm{~ms}$, SD $46.9 \mathrm{~ms}$; SST: mean $505.6 \mathrm{~ms}$, SD $65.1 \mathrm{~ms} ; t=9.240, P<0.001)$. False alarms (commission errors for inhibition trials) were more frequent in stop trials than in no-go trials (GNGT: mean 2.9 (3.6\% of all no-go trials), SD 2.7; SST: mean 8.5 (10.6\% of all stop trials), SD 7.3; $t=3.394, P<0.05)$. This probably reflected a higher $\operatorname{cog}$ nitive load due to more complex instructions in the SST. In both tasks, relatively few misses (commission errors on go trials) occurred (GNGT: mean 1.4 (0.6\% of all go trials), SD, 2.2; SST: mean 1.1 ( $0.5 \%$ of all go trials), SD $2.5 ; t=-0.206, P>0.05)$. As predicted by the independent race model (Verbruggen \& Logan, 2009), in the SST, participants reacted significantly faster on false alarm trials than on successful go trials (false alarm trials: mean $412.5 \mathrm{~ms}$, SD $46.9 \mathrm{~ms}$; successful go trials: mean $505.6 \mathrm{~ms}$, SD $65.1 \mathrm{~ms} ; t=6.042, P=0.000)$.

\section{General linear model analyses}

The GLM analysis of fMRI blood oxygenation level-dependent responses during no-go and stop trials in which inhibition was successful compared with baseline (baseline = fixation; action restraint shown in red, action cancellation shown in blue), their conjunction 

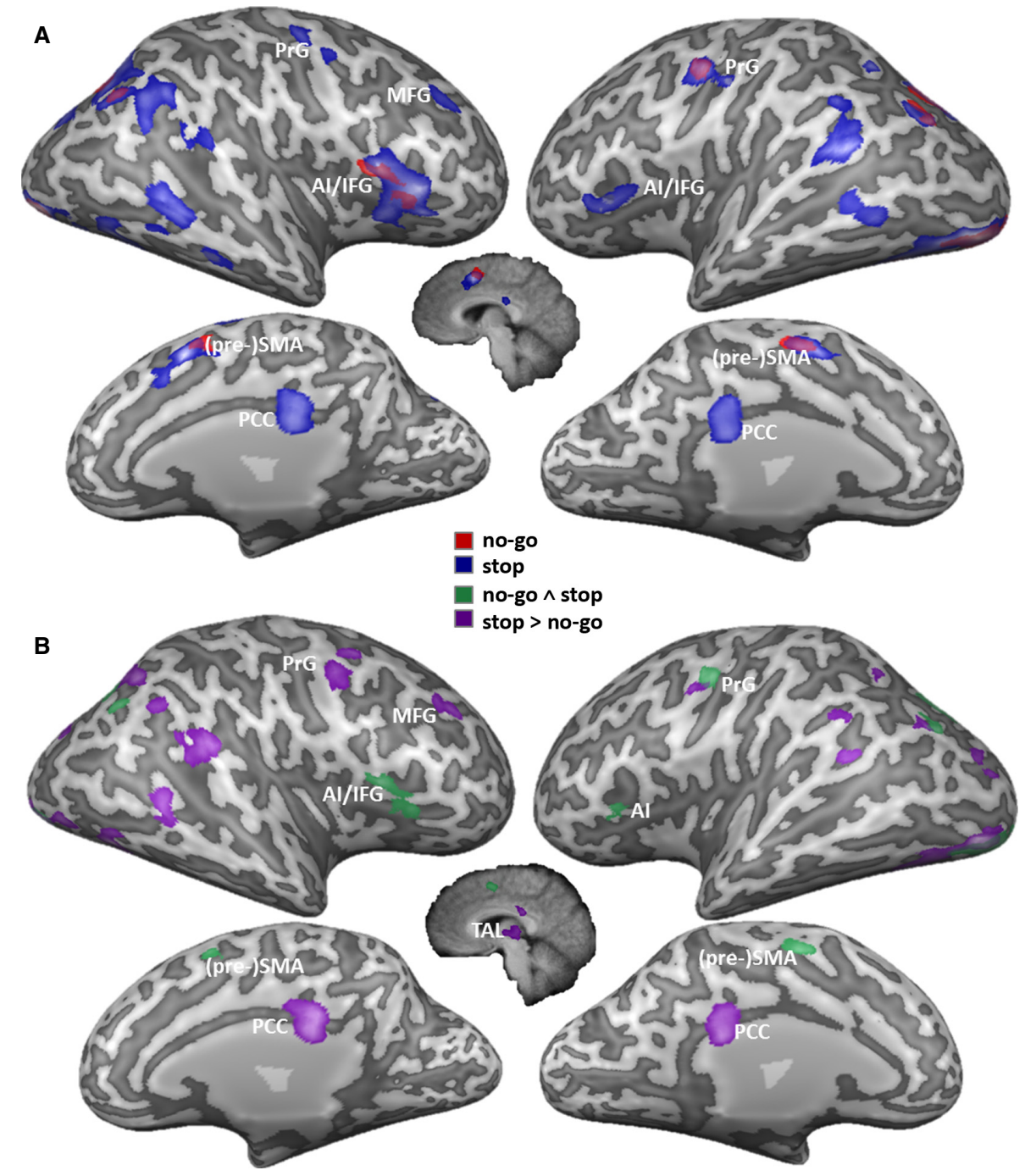

FIG. 2. GLM maps. (A) Increased cortical activation associated with successful inhibition during no-go trials vs. baseline (shown in red, cluster threshold) and stop trials vs. baseline (shown in blue). (B) Conjunction analysis (no-go $\wedge$ stop) (shown in green) and contrast analysis (stop trials $>$ no-go trials) (shown in purple). Statistical maps, $P \leq 0.01$ cluster level threshold corrected (cluster threshold of 28 voxels) random-effects group analysis $(n=17)$. PrG, precentral gyrus; MFG, middle frontal gyrus; AI, anterior insula; TAL, thalamus. Neural activation overlaid over a single representative subject's brain.

(shown in green), and their direct contrast (shown in purple) are shown in Fig. 2 (for Talairach co-ordinates of reported regions see Table 1).

An increase of neural activity during both action restraint and action cancellation (no-go $\wedge$ stop / conjunction) occurred most prominently in the right anterior insula extending into the right inferior frontal gyrus (IFG). In addition, pronounced changes of neural activation in the pre-supplementary and supplementary motor area [(pre-)SMA] were observed. Activation in the left primary motor cortex increased during both no-go and stop trials, mirroring the strong urge to initiate a motor response with the right index finger that was, however, ultimately not completed. Furthermore, activation in parietal and inferior occipital regions increased significantly during both no-go and stop trials.

In addition, during successful action restraint in the GNGT (no-go vs. baseline), the increase of activation in the right superior frontal gyrus was shown to be marginally significant (random-effects analysis, $N=17$, cluster threshold of seven voxels; however, this cluster did not survive the cluster threshold level correction). Furthermore, superior temporal activation increased significantly.
No significant superior frontal activation was found for action cancellation in the SST (stop vs. baseline). During stop trials, activation in the anterior insula (extending into the IFG) bilaterally showed a significant increase, whereby activation was more pronounced in the right inferior frontal regions. Furthermore, activation in the fusiform gyrus increased significantly in successful inhibition trials during SST execution. No such activation was found for the GNGT.

For trials in which inhibition was successful, the direct statistical contrast between both aspects of action inhibition (stop > no-go / direct contrast) revealed no significant increase of the blood oxygenation level-dependent signal in specific brain areas for action restraint (GNGT). For action cancellation (SST), the analysis of the direct contrast revealed SST-specific activation in the right middle frontal gyrus (rMFG), bilateral PCC, and right-lateralized thalamus. Furthermore, activation in bilateral parietal and temporal areas as well as the inferior occipital gyrus was more pronounced for action cancellation.

Based on the described analyses and the hypothetical network suggested by Swick et al. (2011), three ROIs were defined as seed regions for functional connectivity analyses (see section on Connectivity analysis, choice and definition of seed regions above): 
TABLE 1. Talairach co-ordinates for regions activated in different analyses

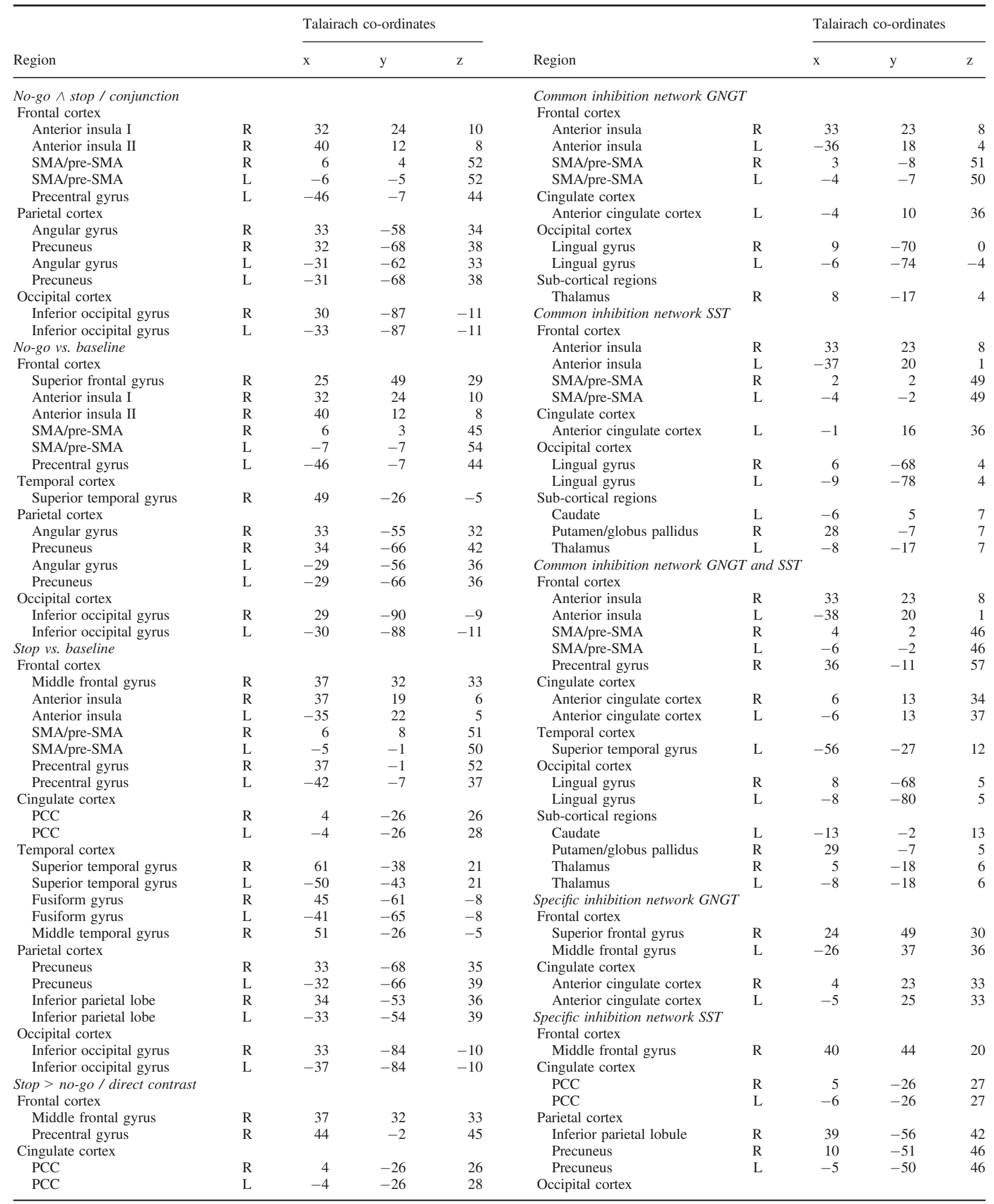


Table 1 (continued)

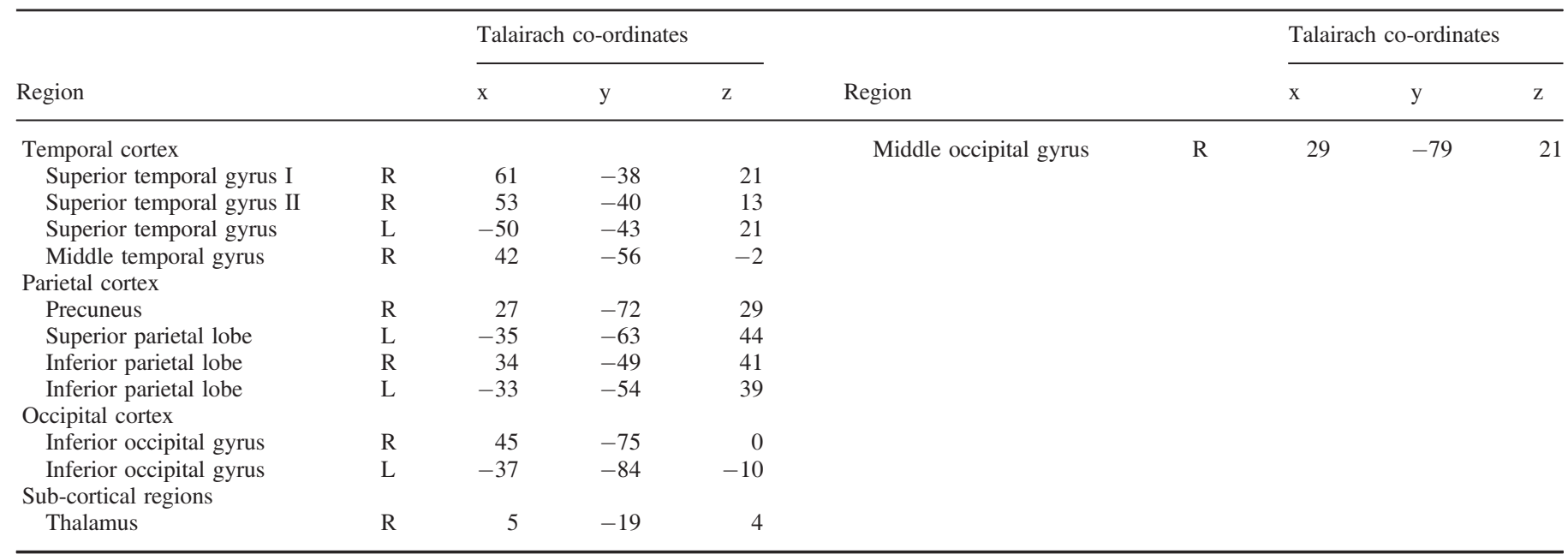

R, right; L, left; SMA, supplementary motor area.

a common ROI in the right anterior insula (Talairach co-ordinates: $\mathrm{x} 33, \mathrm{y} 23, \mathrm{z} 8)$ in order to find evidence for a common inhibition network; an action restraint-specific ROI in the right superior frontal gyrus (Talairach co-ordinates: $\mathrm{x} 24, \mathrm{y} 49, \mathrm{z} 30$ ) in order to find evidence for an action restraint-specific fronto-parietal network; and an action cancellation-specific ROI in the PCC (Talairach co-ordinates: $\mathrm{x} 0, \mathrm{y}-26, \mathrm{z} 26)$ in order to find evidence for an action cancellationspecific cingulo-opercular network.

\section{Functional connectivity analyses}

In addition to the standard GLM fMRI analysis, we conducted functional connectivity analyses in order to enable the specification of overlapping and differential functional networks activated by different aspects of action inhibition (Table 1, Fig. 3). Instantaneous correlation analyses showed that, for both aspects of inhibitory processing, a network including bilateral inferior frontal regions, the (pre-)SMA, anterior cingulate cortex, thalamus, and occipital areas was activated. This common inhibition network was found for the aggregated data of successful no-go and stop trials (Fig. 3, shown in green) and also independently for both paradigms (Fig. 3, action restraint shown in red, action cancellation shown in blue). Although visual inspection of the instantaneous correlation maps suggested that sub-cortical areas might be more strongly involved in the common inhibition network for action cancellation, the direct contrast of the found network for action restraint and action cancellation (connectivity within the common network during stop trials $>$ connectivity within the common network during no-go trials) did not reveal any statistically significant differences in neural activation.

In addition to the common inhibition network, functional connectivity measures showed that, during action restraint, a network including right superior frontal regions, left middle frontal regions, and the anterior cingulate cortex was activated (Fig. 3, shown in red). No such functional network was found during action cancellation. In contrast, for action cancellation, the PCC, rMFG, parietal and occipital regions were found to be functionally related (Fig. 3, shown in blue). No such network was found to be activated during action restraint.

\section{Discussion}

Employing a within-subject within-session event-related fMRI design, analysing functional network connectivity as well as local activity changes, we investigated to what extent the neural correlates of two different aspects of response inhibition, action restraint and action cancellation, rely on a common and/or task-dependent network. We expected to find a common inhibition system within the brain as well as different activation patterns and neural networks involved in action restraint and action cancellation. Specifically, we expected action restraint to activate a task-specific fronto-parietal network and action cancellation to activate a task-specific cinguloopercular network.

\section{A common inhibition network and its lateralization}

\section{A common inhibition network}

In accordance with our hypotheses, we found right and left anterior insula regions, which extend into the IFG, and (pre-)SMA to be activated during successful inhibition in both action restraint (assessed by the GNGT) and action cancellation (assessed by the SST). Our connectivity analyses, moreover, showed task-related functional connectivity between bilateral inferior frontal regions, (pre-)SMA, and thalamic regions. Therefore, our data support the notion that both paradigms share a common pattern of neural activation. We interpret these findings as direct evidence for a common, or core task set, inhibition network across different modalities of response inhibition, as suggested previously (Swick et al., 2011). These brain regions have previously been investigated and associated with inhibitory processing in neuroimaging studies (reviewed in Chambers et al., 2009 and Swick et al., 2011), as well as human lesion (Aron et al., 2003), electroencephalography (Enriquez-Geppert et al., 2010), and brain stimulation (Chambers et al., 2006, 2007; Majid et al., 2012) research.

During successful inhibition, we find an interaction between inferior frontal regions, the (pre-)SMA, and thalamus. This connects to work by Aron (2011) who presented a hypothetical network account suggesting that such an IFG-SMA-thalamus network is involved in global reactive stopping. For the SST, Duann et al. (2009) investigated directed connectivity by means of Granger causality mapping. They found reciprocal connectivity between the inferior frontal cortex and (pre-)SMA. Furthermore, they showed that the (pre-)SMA mediates response inhibition through its connectivity with the primary motor cortex via the basal ganglia circuitry, whereas the inferior frontal cortex interconnects to this system indirectly through its 


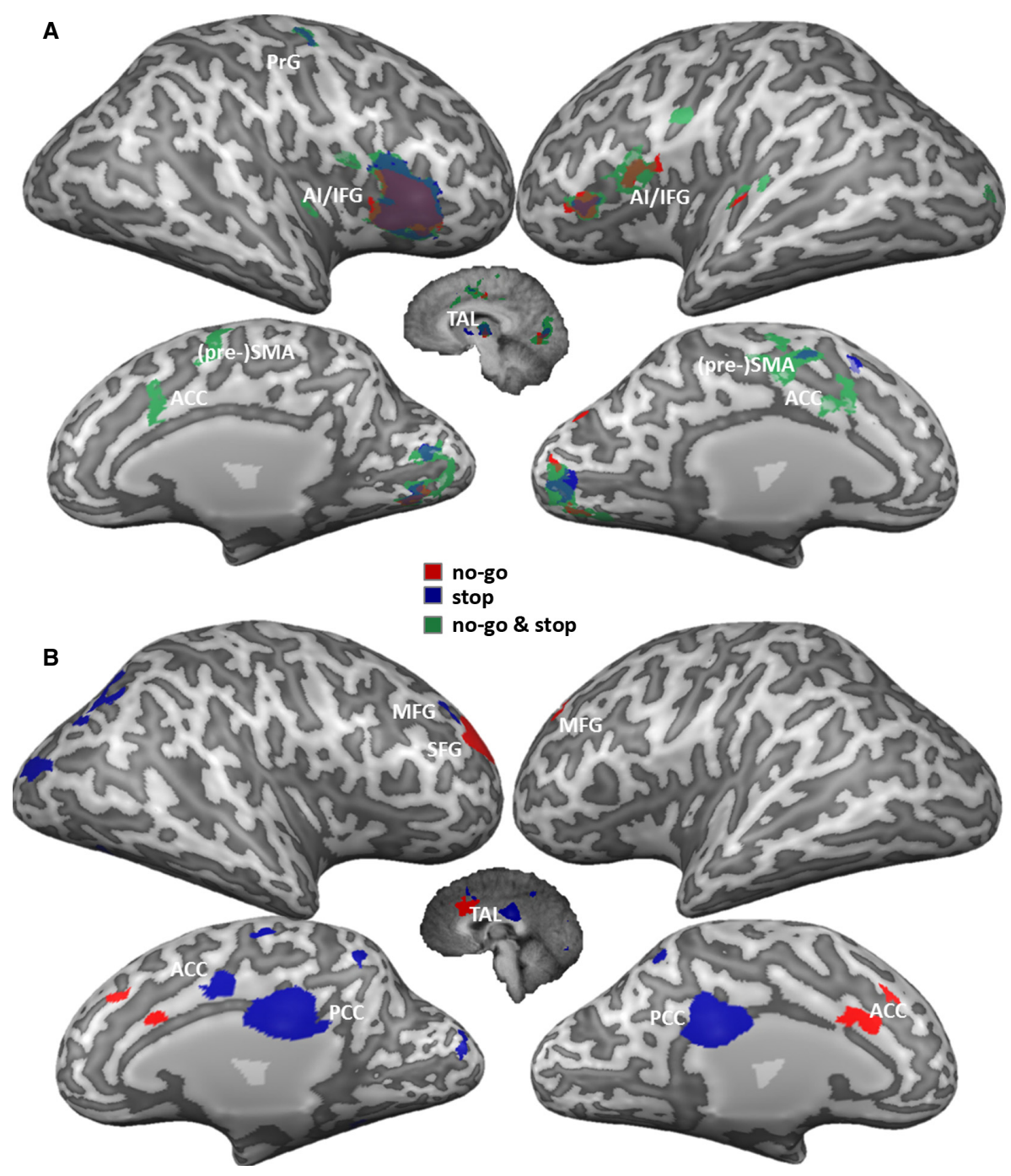

FIG. 3. Functional connectivity within inhibitory networks activated during successful inhibition. (A) Common inhibition network for conjunction of no-go and stop trials shown in green, common network for GNGT shown in red [seed region right anterior insula (AI)]; common network for SST shown in blue (seed region right AI). (B) Specific network for GNGT shown in red [seed region right superior frontal gyrus (SFG)]; specific network for SST shown in blue (seed region PCC). Statistical maps, $P \leq 0.0001$ cluster level threshold corrected (cluster threshold of five voxels) random-effects group analysis $(n=17)$. PrG, precentral gyrus; ACC, anterior cingulate cortex; TAL, thalamus; MFG, middle frontal gyrus; PCC, posterior cingulate gyrus. Neural activation overlaid over a single representative subject's brain.

connectivity with the (pre-)SMA. Recently, Zandbelt et al. (2013) revealed interactions between the inferior frontal cortex, pre-supplementary motor area, striatum and motor cortex using an approach combining repetitive transcranial magnetic brain stimulation and fMRI.

During successful inhibition, inferior frontal regions seem to interact not only with the (pre-)SMA but also with the thalamus. Previous literature also emphasized thalamic involvement in an inhibition network with respect to the anatomical setup of connecting fibre tracts (Aron et al., 2007). Such involvement was mainly shown to work via suppression mechanisms, i.e. thalamic activation should be released during the execution of motor action, whereas it should be suppressed during its inhibition (Aron et al., 2011; Duann et al., 2009). The current findings emphasize that thalamic regions play a crucial role in the processing of motor responses and their inhibition. However, it has to be considered that mere functional connectivity measures cannot enable any interpretation regarding the directivity of such an involvement. More elaborate studies employing directed connectivity measures are needed to clarify the precise interplay of involved regions within a global inhibition network across different inhibitory modalities.

It is of note that the described common inhibition network seems to be crucial not only for the SST but for both tasks independently and also for their conjunction. Future research may therefore need to consider that different response inhibition paradigms show significant overlap in their neural networks. Furthermore, it would be of interest to investigate how action restraint and cancellation are conceptually equivalent to reactive inhibition or also incorporate additionally proactive components (Zandbelt \& Vink, 2010; Zandbelt et al., 2011, 2013a, 2013b) and whether the global inhibition network that we find transfers to these other conceptualizations of action inhibition.

The current study found pronounced activation of inferior frontal regions during successful inhibition for both action restraint and action cancellation. However, although this activation extends into the IFG, our findings indicate that the statistical 'hotspot' for successful inhibition clearly lies in the anterior insula rather than the IFG. This finding implies that the IFG and anterior insula may form 
a functional entity during inhibitory processing, and that the anterior insula may be the most crucial brain region for different forms of response inhibition. This is in contrast to the consistent emphasis on the role of the right IFG in the response inhibition literature (reviewed in Aron et al., 2004), which has been described as being a core region for response inhibition that is involved across different response modalities (Chikazoe et al., 2007). However, in line with the current findings, Swick et al. (2011) found the most prominent overlap of inferior frontal activation to be located in the anterior insula in their meta-analysis of 42 studies of GNGT and SST. They suggest two possible reasons for the disagreement over the IFG or anterior insula as the focus of activation during response inhibition. First, the close proximity of the IFG and anterior insula to each other could result in mislabelling of fMRI activation in either the IFG or anterior insula. Second, extensive spatial smoothing of functional imaging data could blur distinct regions in such a way that activation in the IFG would be smoothed into the anterior insula and vice versa. Swick et al. (2011) suggest that it is especially likely that activation foci in group level statistical maps would appear in the anterior insula. However, despite the strength of this argument, in our data we see the strongest activation focus for both tasks in the anterior insula, even for single subject and unsmoothed group data. This emphasis on the role of the anterior insula alongside IFG activation in response inhibition research follows the example of Sharp et al. (2010), who labeled their focus of activation as the 'IFG/insula', and acknowledges the importance of the anterior insula in inhibition processing and its relation to the IFG. It would be of interest to examine whether these two regions could be functionally discriminated in the context of inhibition research.

\section{Lateralization of the common inhibition network}

We find stronger right hemispheric activation in the bilateral areas of interest and also a greater number of right hemisphere unilateral regions of activation for both tasks. Therefore, our findings suggest that the brain regions involved in response inhibition tasks are asymmetrically distributed towards the right hemisphere. This is in line with most response inhibition literature, independent of the paradigm used (Aron et al., 2004; Chambers et al., 2009; Swick et al., 2011). The evidence presented here is in contrast to the notion of Rubia et al. (2001) that a GNGT would show greater involvement of the left hemisphere, whereas an SST would involve more right hemispheric regions.

Despite the clear right lateralization of activation, our findings also show that brain activation is not exclusively located in the right hemisphere, indicating a role for the left hemisphere in inhibitory processing. Several other studies have shown explicit involvement of the left IFG/left anterior insula in inhibition tasks (Swick et al., 2008; Boehler et al., 2010), suggesting that left hemispheric regions play a role in response inhibition, although to a lesser extent. Therefore, future investigations are needed to clarify the specific division of labour between the two hemispheres for action inhibition.

\section{Distinct neural networks underlying action restraint and action cancellation}

\section{Neural correlates of action restraint}

Contrary to our prediction, the direct contrast between stop trials in the SST and no-go trials in the GNGT does not show any significant differential activation for action restraint (as assessed by the GNGT). We do not find specific GNGT-related increases of neural activation in the rMFG and right inferior parietal lobule/precuneus, as would be expected based on the meta-analysis by Swick et al. (2011). The lack of specific neural activation related to action restraint compared with action cancellation in our data is in line with the findings of Sebastian et al. (2013). They attribute this zero finding to the fact that they presented action restraint trials interleaved with inference-inhibition and action cancellation trials. However, we find the same results using two entirely unrelated (timewise) tasks. Therefore, the question arises whether or not action restraint or withholding might be a sub-form of action cancellation activating no or very few specific neuronal components additional to a global inhibition system.

On a more descriptive level, we find a prefrontal cluster of activation slightly superior to the rMFG around Brodmann area 10 in the GLM analysis of successful no-go trials vs. baseline that we do not find for action cancellation. The functional connectivity that we find between right superior frontal regions, left middle frontal regions, and the anterior cingulate cortex emphasizes the role of these frontal regions for cognitive processing during action restraint. However, we fail to find convincing empirical evidence in favour of a GNGTspecific fronto-parietal network as suggested by Swick et al. (2011). We find some frontal areas as being marginally important for GNGT execution, but no indication of their task-specific connectivity to parietal regions.

In our data, only superior frontal activation was specifically marginally significant for action restraint. However, in the response inhibition literature there is still no consensus on which specific middle and/or superior frontal cortical structures are crucial for inhibitory processing. Simmonds et al. (2008) reported a superior frontal region (Brodmann area 10) similar to the one that we find together with Brodmann area 9 in their activation likelihood estimation over 11 studies investigating the GNGT. Swick et al. (2011) reported, as a result of their activation likelihood estimation over 21 studies investing the GNGT, a middle frontal area (Brodmann area 9) exclusively as the most crucial specific prefrontal area activated during the GNGT. The role of middle and superior frontal areas for inhibition is still not clearly defined and this might motivate a closer look into the precise distribution of specific neural activity in prefrontal areas especially during action restraint.

\section{Neural correlates of action cancellation}

For action cancellation (as assessed by the SST), we find a well-pronounced task-specific neural activation pattern. Hence, in addition to the common network of inhibition described above, action cancellation specifically activates the PCC and thalamic regions in the direct contrast analysis between SST and GNGT. These regions have been associated with functions such as task set control, salience monitoring, and performance monitoring (reviewed in Swick et al., 2011). Furthermore, our data show increased activity in right middle frontal and bilateral parietal areas specifically for action cancellation. This activation pattern could suggest the involvement of a right-lateralized fronto-parietal neural component similar to the dorsal attentional system (Corbetta \& Shulman, 2002) or the executive control system (Seeley et al., 2007), both of which include top-down adaptive cognitive control as one of their central functions. Higher false alarm rates and longer reaction times measured during the SST compared with the GNGT (see behavioural results) suggest that the SST might call for a significantly higher cognitive load or might simply be more difficult. This interpretation could also account for the larger and more pronounced task-related neural activation that we find for the SST compared with the GNGT. 
In our study, a specific task design was employed to optimally compare the two paradigms. To do so, a fixed stop-signal delay was used in the SST. This resulted in significantly longer reaction times in SST compared with GNGT performance and one could assume that participants just waited for the stop signal and thereby transformed the SST simply into a slower GNGT. However, the significantly higher error rate in the SST as well as the fact that we found differential neural activity and networks underlying both paradigms might speak against this interpretation. Nonetheless, it would be of value to develop similar designs for GNGT and SST that still allow for an implementation of variable stop-signal delays in order to gain an even more defined assessment of the underlying differential mechanisms.

Our functional connectivity analysis reveals that, during successful inhibition of stop trials, activity in the PCC is not correlated with activity in thalamic regions as predicted, but rather with the rMFG, parietal, and occipital regions. Instead of being task-specific for action cancellation, our data thus suggest that thalamic regions are an integral component of a common inhibition network relevant during both action cancellation and action restraint. Therefore, the notion of a cingulo-opercular network activated in the stop-signal paradigm is supported by our data on a descriptive level, but not with respect to functional connectivity.

\section{Limitations}

When interpreting our findings, it is important to emphasize that the question of how to label processes underlying the stopping of certain actions is not yet resolved. The label 'inhibition' may often not be an adequate description of the function of a brain region found to be active during response inhibition paradigms.

In the current study, task-related neural activity and functional connectivity were assessed by looking at activity changes during nogo and stop events and their contrasts compared with baseline. Therefore, we cannot interpret our findings as inhibition-specific, but rather as task-specific. Claims can be made concerning the common and distinct networks recruited for the two tasks, but specificity to inhibitory processes cannot be concluded based solely on these results. It is debatable which contrast to look at for inhibition-specific activity (Swick et al., 2011) and additional options to the comparison against baseline would be to compare successful inhibition with false alarms or go events. Our design did not provide a sufficient number of false alarm trials to allow such statistical comparisons. Directly contrasting no-go and stop events with go events, however, did not reveal any significant results. This might be due to power issues. Essentially, we had to focus on the comparison to baseline, which has to be seen as a rather sub-optimal choice of contrasts in response inhibition research (Swick et al., 2011). Our hypotheses focused on a task comparison and, thus, on differential task-related activity during inhibitory processing rather than the isolation of one specific form of inhibition. Furthermore, the type of connectivity analysis employed investigates neural activity during a certain time window rather than in contrast to other time courses. Therefore, the choice to base our analyses on the comparison against baseline is justified. However, it has to be emphasized that the activation found might not be specific to action restraint or action cancellation, but could reflect other processes (e.g. response preparation). Further investigations have to clarify how far the results bear up to a more specific choice of contrasts.

Furthermore, there are several considerations to take into account when thinking about assigning certain functions to the involved regions. Although (pre-)SMA and thalamic regions seem to be specifically involved in the mechanisms underlying the withdrawal of motor action (reviewed in Swick et al., 2011), the exact role of inferior frontal brain structures is not clear. Variations in the task design of go/no-go and stop-signal paradigms as well as the addition of control trials that can tap into processes such as action selection or the choice of alternative action plans may be specifically capable of shedding light on this important question. For example, Verbruggen et al. (2010) showed that the disruption of neural tissue in the ventral right IFG by theta-burst transcranial magnetic brain stimulation did affect both stop-signal trials in which subjects had to withhold their response and dual-signal trials in which subjects had to execute an additional response. This finding suggests that, when the cognitive system finds itself confronted with alternative competing plans, inferior frontal regions might serve as a higher-order control of updating and selecting the respective action plans, rather than specifically underlying their simple inhibition. Schall \& Godlove (2012) point out that, when taking into account studies not focusing on inhibition only but also other cognitive processes, the function of the inferior frontal cortex might rather be labeled as attention capture modulating with stimulus unexpectedness. These described functions of inferior frontal activity are similar to those discussed with regard to the anterior insula. Although classically understood as a tool of interoceptive awareness (Craig, 2009), anterior insula functions include the selection of appropriate responses, cognitive control, maintenance of task set, and focal attention (Menon \& Uddin, 2010; Nelson et al., 2010). Other authors have emphasized the role of right inferior frontal regions in attentional capture (Sharp et al., 2010) and attentional control (Hampshire et al., 2010). All of these aspects are crucial for successful inhibitory processing of any kind.

Due to this evidence, we cannot conclusively label the function of the brain regions that we find to be associated with action restraint and action cancellation as 'inhibition'. This is partly also to be attributed to the limitations of using fMRI as a rather coarse method to study brain activation related to inhibition. Ultimately, the spatial resolution of imaging methods has to be combined with a more precise approach time-wise. For instance, in the context of SST research, Schall \& Godlove (2012) emphasized the importance of looking at the modulation of activity in certain brain regions with respect to time components inherent to the task (e.g. stop-signal reaction time in the SST). Further research is needed to clarify the detailed allocation of tasks between involved brain structures in inhibitory processing. However, activation in inferior frontal brain areas, in particular, is certainly an integral component of every process associated with inhibition. Therefore, this activation seems to be fundamental to response inhibition.

\section{Conclusion and implications}

On a behavioural level, action restraint and action cancellation share the task instruction not to respond to a certain configuration of stimuli. Our findings show that they rely on a common network of neural activation including bilateral inferior frontal regions, the (pre-) SMA, and thalamic regions. However, our findings also point to additional neural components that are distinct to action restraint (i.e. the right superior frontal gyrus and anterior cingulate cortex) or action cancellation (i.e. the rMFG and PCC). We therefore conclude that response inhibition-related neural activity across different inhibitory aspects does refer to a common inhibition system within the brain, but is in part also task dependent.

It might be that the strict dichotomous view of action restraint vs. action cancellation as two independent dimensions of action inhibi- 
tion needs revision. Instead of being conceptualized dichotomously, the two concepts might rather combine independent inhibitory processing with a fundamental shared cognitive inhibition system. Our findings of common, as well as specific, inhibitory neural components would support such a claim.

The findings of the present study suggest that the decision to use specific inhibition tasks in future neuroscientific research should be based on theoretical considerations that take into account the conceptual as well as the neural aspects of the hypotheses to be investigated. Furthermore, it is clear that, in clinical settings, the choice to include response inhibition paradigms as elements in diagnostic batteries should be made carefully. Therefore, with respect to the diagnostics of specific clinical disorders, it should be carefully reviewed which of the distinct inhibition aspects are relevant. In this way, research like this can contribute to disorder-specific (or even criteria-specific) tailored assessment and (perhaps in the very far future) alteration or treatment.

\section{Acknowledgements}

This work was supported by a grant from the Netherlands Organization for Scientific Research (NWO; HCMI 10-19). We thank Katherine L. Wheat, Armin Heinecke, and Thomas C. Emmerling for their helpful support.

\section{Abbreviations}

fMRI, functional magnetic resonance imaging; GLM, general linear model; GNGT, go/no-go task; IFG, inferior frontal gyrus; PCC, posterior cingulate cortex; (pre-)SMA, pre-supplementary motor area; rMFG, right middle frontal gyrus; ROI, region of interest; SST, stop-signal task.

\section{References}

Aron, A.R. (2011) From reactive to proactive and selective control: developing a richer model for stopping inappropriate responses. Biol. Psychiat., 69, e55-e68.

Aron, A.R., Fletcher, P.C., Bullmore, E.T., Sahakian, B.J. \& Robbins, T.W. (2003) Stop-signal inhibition disrupted by damage to right inferior frontal gyrus in humans. Nat. Neurosci., 6, 115-116.

Aron, A.R., Robbins, T.W. \& Poldrack, R.A. (2004) Inhibition and the right inferior frontal cortex. Trends Cogn. Sci., 8, 170-177.

Aron, A.R., Behrens, T.E., Smith, S., Frank, M.J. \& Poldrack, R.A. (2007) Triangulating a cognitive control network using diffusion-weighted magnetic resonance imaging (MRI) and functional MRI. J. Neurosci., 27, 3743-3752.

Boehler, C.N., Appelbaum, L.G., Krebs, R.M., Hopf, J.M. \& Woldorff, M.G. (2010) Pinning down response inhibition in the brain conjunction analyses of the Stop-signal task. NeuroImage, 52, 1621-1632.

Chambers, C.D., Bellgrove, M.A., Stokes, M.G., Henderson, T.R., Garavan, H., Robertson, I.H., Morris, A.P. \& Mattingley, J.B. (2006) Executive "brake failure" following deactivation of human frontal lobe. J. Cognitive Neurosci., 18, 444-455.

Chambers, C.D., Bellgrove, M.A., Gould, I.C., English, T., Garavan, H., McNaught, E., Kamke, M. \& Mattingley, J.B. (2007) Dissociable mechanisms of cognitive control in prefrontal and premotor cortex. J. Neurophysiol., 98, 3638-3647.

Chambers, C.D., Garavan, H. \& Bellgrove, M.A. (2009) Insights into the neural basis of response inhibition from cognitive and clinical neuroscience. Neurosci. Biobehav. Rev., 33, 631-646.

Chikazoe, J., Konishi, S., Asari, T., Jimura, K. \& Miyashita, Y. (2007) Activation of right inferior frontal gyrus during response inhibition across response modalities. J. Cognitive Neurosci., 19, 69-80.

Corbetta, M. \& Shulman, G.L. (2002) Control of goal-directed and stimulusdriven attention in the brain. Nat. Neurosci., 3, 201-215.

Craig, A.D. (2009) How do you feel - now? The anterior insula and human awareness. Nat. Neurosci., 10, 59-70.

Deichmann, R., Gottfried, J.A., Hutton, C. \& Turner, R. (2003) Optimized EPI for fMRI studies of the orbitofrontal cortex. Neurolmage, 19, $430-441$.
Duann, J.R., Ide, J.S., Luo, X. \& Li, C.S. (2009) Functional connectivity delineates distinct roles of the inferior frontal cortex and presupplementary motor area in stop signal inhibition. J. Neurosci., 29, 1017110179.

Eagle, D.M., Bari, A. \& Robbins, T.W. (2008) The neuropsychopharmacology of action inhibition: cross-species translation of the stop-signal and go/ no-go tasks. Psychopharmacology, 199, 439-456.

Enriquez-Geppert, S., Konrad, C., Pantev, C. \& Huster, R.J. (2010) Conflict and inhibition differentially affect the N200/P300 complex in a combined go/no-go and stop-signal task. Neurolmage, 51, 877-887.

Forman, S.D., Cohen, J.D., Fitzgerald, M., Eddy, W.F., Mintun, M.A. \& Noll, D.C. (1995) Improved assessment of significant activation in functional magnetic resonance imaging (fMRI), use of a cluster-size threshold. Magn. Reson. Med., 33, 636-647.

Goebel, R., Roebroeck, A., Kim, D.S. \& Formisano, E. (2003) Investigating directed cortical interactions in time-resolved fMRI data using vector autoregressive modeling and Granger causality mapping. Magn. Reson. Imaging, 21, 1251-1261.

Hampshire, A., Chamberlain, S.R., Monti, M.M., Duncan, J. \& Owen, A.M. (2010) The role of the right inferior frontal gyrus: inhibition and attentional control. Neurolmage, 50, 1313-1319.

Logan, G.D., Schachar, R.J. \& Tannock, R. (1997) Impulsivity and inhibitory control. Psychol. Sci., 8, 60-64.

Majid, D.S.A., Cai, W., George, J.S., Verbruggen, F. \& Aron, A.R. (2012) Transcranial magnetic stimulation reveals dissociable mechanisms for global versus selective corticomotor suppression underlying the stopping of action. Cereb. Cortex, 22, 363-371.

McNab, F., Leroux, G., Strand, F., Thorell, L., Bergman, S. \& Klingberg, T. (2008) Common and unique components of inhibition and working memory: an fMRI, within-subjects investigation. Neuropsychologia, 46, $2668-2682$.

Menon, V. \& Uddin, L.Q. (2010) Saliency, switching, attention, and control: a network model of insula function. Brain Struct. Funct., 214, 655-667.

Nelson, S.M., Dosenbach, N.U., Cohen, A.L., Wheeler, M.E., Schlaggar, B.L. \& Petersen, S.E. (2010) Role of anterior insula in task-level control and focal attention. Brain Struct. Funct., 214, 669-680.

Roebroeck, A., Formisano, E. \& Goebel, R. (2005) Mapping directed influence over the brain using Granger causality and fMRI. NeuroImage, 25, 230-242.

Rubia, K., Russell, T., Overmeyer, S., Brammer, M.J., Bullmore, E.T., Sharma, T., Simmons, A., Williams, S.C.R., Giampietro, V., Andrew, C.M. \& Taylor, E. (2001) Mapping motor inhibition: conjunctive brain activations across different versions of go/no-go and stop tasks. Neurolmage, 13, 250-261.

Schachar, R., Logan, G.D., Robaey, P., Chen, S., Ickowicz, A. \& Barr, C. (2007) Restraint and cancellation: multiple inhibition deficits in attention deficit hyperactivity disorder. J. Abnorm. Child Psych., 35, 229-238.

Schall, J.D. \& Godlove, D.C. (2012) Current advances and pressing problems in studies of stopping. Curr. Opin. Neurobiol., 22, 1012-1021.

Sebastian, A., Pohl, M.F., Klöppel, S., Feige, B., Lange, T., Stahl, C., Voss, A., Klauer, K.C., Lieb, K. \& Tüscher, O. (2013) Disentangling common and specific neural subprocesses of response inhibition. NeuroImage, 64, 601-615.

Seeley, W.W., Menon, V., Schatzberg, A.F., Keller, J., Glover, G.H., Kenna, H., Reiss, A.L. \& Greicius, M.D. (2007) Dissociable intrinsic connectivity networks for salience processing and executive control. J. Neurosci., 27, 2349-2356.

Sharp, D.J., Bonnelle, V., De Boissezon, X., Beckmann, C.F., James, S.G., Patel, M.C. \& Mehta, M.A. (2010) Distinct frontal systems for response inhibition, attentional capture, and error processing. Proc. Natl. Acad. Sci. USA, 107, 6106-6111.

Simmonds, D.J., Pekar, J.J. \& Mostofsky, S.H. (2008) Meta-analysis of Go/No-go tasks demonstrating that fMRI activation associated with response inhibition is task-dependent. Neuropsychologia, 46, 224232.

Swick, D., Ashley, V. \& Turken, A.U. (2008) Left inferior frontal gyrus is critical for response inhibition. BMC Neurosci., 9, 1471-2202.

Swick, D., Ashley, V. \& Turken, U. (2011) Are the neural correlates of stopping and not going identical? Quantitative meta-analysis of two response inhibition tasks. NeuroImage, 56, 1655-1665.

Talairach, J. \& Tournoux, P. (1988) Co-Planar Stereotaxic Atlas Of The Human Brain: An Approach To Medical Cerebral Imaging. Thieme, New York.

Verbruggen, F. \& Logan, G.D. (2009) Models of response inhibition in the stopsignal and stop-change paradigms. Neurosci. Biobehav. Rev., 33, 647-661. 
Verbruggen, F., Aron, A.R., Stevens, M.A. \& Chambers, C.D. (2010) Theta burst stimulation dissociates attention and action updating in human inferior frontal cortex. Proc. Natl. Acad. Sci. USA, 107, 13966-13971.

Wöstmann, N.M., Aichert, D.S., Costa, A., Rubia, K., Möller, H.J. \& Ettinger, U. (2012) Reliability and plasticity of response inhibition and interference control. Brain Cogn., 81, 82-94.

Zandbelt, B.B. \& Vink, M. (2010) On the role of the striatum in response inhibition. PLOS ONE, 5, e13848.

Zandbelt, B.B., van Buuren, M., Kahn, R.S. \& Vink, M. (2011) Reduced proactive inhibition in schizophrenia is related to corticostriatal dysfunction and poor working memory. Biol. Psychiat., 70, 1151-1158.
Zandbelt, B.B., Bloemendaal, M., Neggers, S.F.W., Kahn, R.S. \& Vink, M. (2013a) Expectations and violations: delineating the neural network of proactive inhibitory control. Hum. Brain Mapp., 34, 20152024.

Zandbelt, B.B., Bloemendaal, M., Hoogendam, J.M., Kahn, R.S. \& Vink, M. (2013b) Transcranial magnetic stimulation and functional MRI reveal cortical and subcortical interactions during stop-signal response inhibition. $J$. Cognitive Neurosci., 25, 157-174.

Zheng, D., Oka, T., Bokura, H. \& Yamaguchi, S. (2008) The key locus of common response inhibition network for no-go and stop signals. J. Cognitive Neurosci., 20, 1434-1442. 